\title{
Autochthonous Oenococcus oeni Strain to Avoid Histamine Formation in Red Wines: A Study in Real Winemaking Conditions
}

\author{
Silvia Pérez-Magariño, ${ }^{1 *}$ Estela Cano-Mozo, ${ }^{1}$ Clara Albors,${ }^{2}$ Antonio Santos, ${ }^{3}$ \\ and Eva Navascués ${ }^{2}$
}

\begin{abstract}
The production of wines with low biogenic amine (BA) concentrations is a current concern in the wine sector, and strategies to avoid the formation of BAs during winemaking are of particular interest. The aim of this work was to determine the influence of selected autochthonous Oenococcus oeni lactic acid bacteria (LAB) on the BA content in red wines and their prevalence against the indigenous microbiota to avoid BA formation. Sixty-seven red wines were produced at industrial scale under real winemaking conditions for three consecutive vintages. For each wine, we determined LAB implantation and the BA concentrations at various stages of the winemaking process. The results clearly indicated that the use of selected $O$. oen $i$ strains that are unable to produce BA, in combination with adapted biomass production, is a good strategy to control histamine production in wines. These practices, carried out over three consecutive years, were also observed to ensure the persistence of the selected autochthonous O. oeni strain (CECT 9749) against other indigenous microbiota in the entire winery. Furthermore, analysis of BA content during wine aging in barrels indicated that low BA content was maintained, resulting in healthier wines for the consumer.
\end{abstract}

Keywords: autochthonous strains, biogenic amines, histamine, lactic acid bacteria, malolactic fermentation, Oenococcus oeni

Biogenic amines (BAs) are low-molecular-weight, organic nitrogenous compounds that occur naturally in fermented foods and wines (Gardini et al. 2016). Under normal physiological conditions, BAs are metabolized by gastrointestinal enzymes (Gardini et al. 2016, Ancín-Azpilicueta et al. 2019). However, the consumption of large amounts of BAs could have adverse effects on consumers' health from a toxicological point of view (EFSA 2011). Histamine and tyramine are considered the most toxic amines, causing negative effects such as headaches, nausea, hypo- and hypertension, respiratory disorders, tachycardia, and various allergic disorders

\footnotetext{
${ }^{1}$ Instituto Tecnológico Agrario de Castilla y León. Consejería de Agricultura y Ganadería. Ctra Burgos Km 119, Finca Zamadueñas. 47071 Valladolid, Spain; ${ }^{2}$ Pago de Carraovejas State Winery. Camino de Carraovejas, s/n. 47300 Peñafiel, Valladolid, Spain; and ${ }^{3}$ Department of Genetics, Physiology and Microbiology. Unit of Microbiology. Biology Faculty, Complutense University of Madrid, Madrid, Spain.

*Corresponding author: (permagsi@itacyl.es; tel: +34 983 415245)

Acknowledgments: This study is part of HEALTHWINE Project and GLOBALVITI Project (IDI-20160746 with the financial support of the CDTI-CIEN program). The authors would like to thank Agrovin S.A. for technical assistance.

Manuscript submitted Feb 2020, revised March 2020, Sept 2020, accepted Nov 2020

This is an open access article distributed under the CC BY license (https:// creativecommons.org/licenses/by/4.0/).

By downloading and/or receiving this article, you agree to the Disclaimer of Warranties and Liability. The full statement of the Disclaimers is available at http://www.ajevonline.org/content/proprietary-rights-notice-ajev-online. If you do not agree to the Disclaimers, do not download and/or accept this article. doi: 10.5344/ajev.2020.20010
}

(Landete et al. 2005, Ladero et al. 2010, Moreno-Arribas et al. 2010, Gardini et al. 2016).

Some BAs, such as putrescine and cadaverine, are nontoxic polyamines but can potentiate the toxic effects of other BAs through the inhibition of enzymes that detoxify histamine, tyramine, and phenylethylamine (Straub et al. 1995, Özogul and Özogul 2019). In addition, these polyamines adversely affect wine sensory quality (Tomera 1999, García-Villar et al. 2007).

Therefore, the presence of high BA concentrations is related to wine safety (EFSA 2011, Martuscelli et al. 2013) but may also be an indicator of wine quality and hygienic conditions (Del Prete et al. 2009, Gardini et al. 2016).

Currently, the European Union has not established a regulatory limit for histamine or any other BA in wines. However, various countries in Europe have set their own recommended limits for histamine in wine, ranging from 2 to $10 \mathrm{mg} / \mathrm{L}$ (Smit et al. 2008). The International Organisation of Vine and Wine (OIV) recommends not exceeding 12 $\mathrm{mg} / \mathrm{L}$ of histamine.

Estimating safe levels of BAs in wine is difficult because it depends on several factors such as the type of amine, its concentration, the physiological conditions of consumers (whether individuals have mechanisms for degrading these compounds), and the consumption of other BA-containing foods (which could increase their toxicity) (Ancín-Azpilicueta et al. 2019). In addition, it has been demonstrated that ethanol and acetaldehyde may enhance the toxicity of amines in humans through the inhibition of amino oxidase enzymes responsible for amine degradation (Zimatkin and Anichtchik 1999). 
Histamine, putrescine, cadaverine, tyramine, phenylethylamine, and spermidine are the main BAs in wine (Moreno-Arribas and Polo 2009, EFSA 2011). Some BAs, such as putrescine and spermidine, can be present in grapes (Landete et al. 2005, Izquierdo Cañas et al. 2008, Del Prete et al. 2009), but most are produced during winemaking by microorganisms. The formation of BAs requires amino acids, microorganisms with decarboxylase activity, and favorable conditions for the growth of those microorganisms (Smit et al. 2008, Costantini et al. 2009, Moreno-Arribas et al. 2010). Some authors reported that yeasts can produce BAs during alcoholic fermentation (AF) (Ancín-Azpilicueta et al. 2008, Smit et al. 2008) or during wine storage (Jiménez-Moreno et al. 2003, Hernández-Orte et al. 2008). Although reported results are contradictory, it is assumed that the greatest amount of BAs, especially histamine, is produced during malolactic fermentation (MLF) through the decarboxylation activity of lactic acid bacteria (LAB) that transform amino acids into BAs (Soufleros et al. 1998, Lonvaud-Funel 1999, Landete et al. 2005, Marcobal et al. 2006).

Oenococcus oeni is the species of LAB most commonly found after AF both in spontaneous MLF (due to the growth of indigenous strains) and in MLF inoculated with selected strains (Moreno-Arribas et al. 2003, Nehme et al. 2010). This is due to the fact that $O$. oeni is well adapted to the harsh wine conditions (high ethanol, low $\mathrm{pH}$, low nutrient levels, and the presence of $\mathrm{SO}_{2}$ ) (Lonvaud-Funel 1999). Other LAB species of different genera, such as Lactobacillus, Leuconostoc, and Pediococcus, can also grow in wine, especially if $\mathrm{pH}$ values are higher than 3.5 (Lonvaud-Funel 2001, Costantini et al. 2009). The ability of these bacterial species to produce BAs seems to be strain dependent (Berbegal et al. 2017). Therefore, it is important to select LAB strains that do not produce BAs in order to minimize the wine's BA content. Some authors reported that selection of LAB strains without decarboxylase activity can be used to prevent BA formation in wines (Marcobal et al. 2006). Moreover, simultaneous yeast/LAB co-inoculation has been shown to be effective for avoiding BA production and obtaining wines with better sensory characteristics (Massera et al. 2009, Izquierdo Cañas et al. 2015).

The occurrence of BAs in wines has been studied in recent years, and winemakers have been seeking strategies to avoid BA formation during winemaking to obtain wines with low BA concentrations (Benito 2019a). Several strategies have been suggested to control the production of BAs or to degrade them. The use of non-Saccharomyces strains that decrease malic acid content and the use of different retention systems (not used for quality wines) have been studied in depth (Benito 2019b, Rodríguez-Bencomo and Mehdi 2019). Currently, one of the main strategies to prevent the formation of BAs in wines at the industrial scale is the inoculation of selected non-BA-producing $O$. oen $i$ strains (Moreno-Arribas et al. 2003, Izquierdo Cañas et al. 2009). However, the induction of MLF by these commercial LAB was not always successful in a whole winery, due to various factors such as wine being a very harsh medium for LAB growth (Ruiz et al. 2010) and the competitive adaptive advantage of autochthonous micro- biota to the winery conditions. For this reason, some authors have suggested the use of selected autochthonous LAB of a specific wine-producing area, which could improve the MLF development (Ruiz et al. 2010, Berbegal et al. 2017).

Therefore, the aim of this work was to study the influence of selected autochthonous $O$. oeni on the BA content of red wines from Ribera del Duero during three consecutive vintages $(2016,2017$, and 2018) and to study their prevalence against the indigenous microbiota. The importance of managing the adaptation of the LAB culture and how to ensure its presence in winery tanks was studied in order to avoid histamine formation in wines. We also evaluated the effects of $\mathrm{LAB}$ inoculation and wine aging in oak barrels on BA production.

\section{Materials and Methods}

Winemaking conditions. Red wines were produced via the usual winemaking process in Pago de Carraovejas State winery, located in Peñafiel (Valladolid, Spain) within the Ribera del Duero Geographical Indication. Vineyards belonging to the winery are in a valley at elevations between 850 and $950 \mathrm{~m}$ asl and are cultivated under organic farming conditions. The grape variety is a "Tinto Fino" ("Tempranillo") Carraovejas clone with 110R rootstock.

Maturity control was checked using not only technical parameters (sugar, $\mathrm{pH}$, total acidity, weight/100 grape berries) but also quality parameters such as the Glories method (Cromoenos), which evaluates the phenolic maturity of grapes. This provides information on both the quantity (total potential in anthocyanins and tannins) and the quality (anthocyanin extractability, seed maturity) of the polyphenols.

This work, performed during three consecutive harvests, was done with the normal winemaking conditions and practices used at the cellar facilities. Because of differences in the characteristics and grape yield between vintages, the experimental design was slightly different each year. However, to establish a suitable point of comparison, half of the harvest for each vintage was inoculated with the selected $O$. oeni CECT 9749 strain and compared with the other half.

In this study, three consecutive years were compared: 2016 (harvest start/end, 4 Oct/29 Oct), 2017 (harvest start/end, 19 Sept/30 Sept), and 2018 (harvest start/end, 29 Sept/12 Oct). Grapes were harvested manually and immediately transported in $12-\mathrm{kg}$ boxes to the winery where they underwent double selection to avoid any rot or immature clusters. The clusters were then destemmed and lightly crushed.

For all vintages, the alcoholic fermentations were carried out in 25,000-L stainless steel tanks filled with $18,000 \mathrm{~kg}$ of red grapes. Workers collected $396,000 \mathrm{~kg}$ of grapes for the 2016 vintage, $324,000 \mathrm{~kg}$ for the 2017 vintage, and 486,000 kg for the 2018 vintage; 22,18 , and 27 fermentation tanks were filled for each vintage, respectively (Table 1).

AF was carried out with the yeast strain Saccharomyces cerevisiae CECT 12008 (Spanish Type Culture Collection) that was previously isolated and selected from the vineyards of Pago de Carraovejas State winery. Yeast was inoculated during the filling of each tank to a concentration of $10^{6}$ cells $/ \mathrm{mL}$ of must or $10^{6}$ cells $/ \mathrm{mg}$ of grape. In addition, some 
fermentations were spontaneously developed with the indigenous yeasts in each harvest to see the influence of spontaneous AF on the development of the inoculated bacterial culture and/or on the production of BAs.

$\mathrm{AF}$ kinetics studies were conducted at $24^{\circ} \mathrm{C} \pm 1^{\circ} \mathrm{C}$. Temperature (cap and liquid) and sugar content were measured daily during fermentation. $\mathrm{SO}_{2}$ was added at a concentration of $30 \mathrm{mg} / \mathrm{L}$ during the filling of the fermentation tanks. It was supplemented with organic nitrogen without diammonium phosphate (Actimax Natura, Agrovin) at $0.50 \mathrm{~g} / \mathrm{L}$ doses at the beginning of the fermentation. For color extraction, maceration was conducted with fermentation and pumping with aeration twice per day. When fermentation and maceration finished, the wines were devatted and only the first pressings (55\% yield) were taken.

LAB strain and inocula preparation. An autochthonous O. oeni CECT 9749 strain was used to inoculate the experimental wines. This strain was isolated and selected from red wines of the same winery in a previous study (Berbegal et al. 2017). This non-histaminogenic strain was selected because of its prevalence in wines, high alcohol resistance, and high quality properties that do not affect wine fruitiness. In addition, this strain was found to be well adapted to the conditions of these wines, competing effectively with other indigenous LAB strains (Berbegal et al. 2017).

Inoculum production was performed following a strict scale-up procedure to reach $>10^{9} \mathrm{CFU} / \mathrm{mL}$. The selected $O$. oeni CECT 9749 strain was initially grown in MLO broth (Zúñiga et al. 1993) to reach an early stationary phase. Cells were then centrifuged ( $8000 \mathrm{rpm}, 10 \mathrm{~min}$ ), washed with Ringer's solution, and transferred to $10 \mathrm{~mL}$ of culture media at a final concentration of $1 \times 10^{6} \mathrm{CFU} / \mathrm{mL}$. Then, cellular concentration was scaled up through a three-stage procedure $(0.5$, 10 , and $80 \mathrm{~L}$ ) using a liquid production medium described in Berbegal et al. (2015) with several modifications that allowed the culture to adapt to the harsh conditions of red wines: use of red wine with a total polyphenol index above 70 and a higher alcohol level, fermentation activator enhancers as Actimax NATURA (Agrovin S.A.), and cofactors (Mg, Mn, folic acid, ascorbic acid, and retinol).

Table 1 Design of experiments conducted in the present work during three consecutive vintages. The Oenococcus oeni CECT 9749 strain was used in the inoculated tanks.

\begin{tabular}{lccc}
\hline Vintage/feature & $\begin{array}{c}\text { Inoculated } \\
\text { tanks }\end{array}$ & $\begin{array}{c}\text { Non-inoculated } \\
\text { tanks }\end{array}$ & Total \\
\hline $\mathbf{2 0 1 6}$ & & 13 & 22 \\
Number of tanks & 9 & 234,000 & 396,000 \\
Volume (kg) & 162,000 & 59.1 & 100 \\
Percentage (\%) & 40.9 & & \\
$\mathbf{2 0 1 7}$ & & 8 & 18 \\
Number of tanks & 10 & 144,000 & 324,000 \\
Volume (kg) & 180,000 & 44.4 & 100 \\
Percentage (\%) & 55.6 & & \\
$\mathbf{2 0 1 8}$ & & 11 & 27 \\
Number of tanks & 16 & 198,000 & 486,000 \\
Volume (kg) & 288,000 & 40.7 & 100 \\
Percentage (\%) & 59.3 & & \\
\hline
\end{tabular}

The industrial biomass production was performed in a fermenter with $80 \mathrm{~L}$ of production medium under sterile conditions (Bioprocess technology Bio-pro $100 \mathrm{~L}$ ). This was used to inoculate each $25,000-\mathrm{L}$ tank with the selected $O$. oeni strain $\left(2 \times 10^{6} \mathrm{CFU} / \mathrm{mL}\right)$. A microbiological analysis of the three scale-up steps was carried out to certify the correct sterilization of the culture medium (Berbegal et al. 2015). This scale-up was done in Agrovin S.A. facilities.

Inoculation of lactic acid cultures and MLF. The autochthonous selected $O$. oeni CECT 9749 strain was inoculated at the beginning of the AF, ensuring no free $\mathrm{SO}_{2}$. The first tanks of each harvest (2016, 2017, and 2018) were inoculated, with effort made to maintain equal numbers of inoculated and non-inoculated tanks. The percentage of inoculated tanks was $40.9,55.6$, and $59.3 \%$ for each harvest, respectively (Table 1).

Once AF was finished (glucose + fructose content $<1$ $\mathrm{g} / \mathrm{L}), \mathrm{MLF}$ was conducted at $22^{\circ} \mathrm{C} \pm 1{ }^{\circ} \mathrm{C}$ until the malic acid level was $<0.2 \mathrm{~g} / \mathrm{L}$. At this point, MLF was considered to be finished.

Aging in cellar. After MLF, the wines were racked, corrected with $\mathrm{SO}_{2}\left(\sim 0.5\right.$ of molecular $\left.\mathrm{SO}_{2}\right)$, and aged in French oak barrels $(225 \mathrm{~L})$ for 12 months in a cellar with a temperature of $15^{\circ} \mathrm{C}$ and a controlled relative humidity of $\sim 75$ to $85 \%$.

Chemicals. Chromatographic-grade reagents were provided by Carlo Erba Reagents; 2-aminoheptanoic acid (internal standard) and diethylethoxymethylenemalonate (DEEMM) were obtained from Fluka (Sigma-Aldrich); the remaining reagents were supplied by Panreac. Milli-Q-grade water was obtained using a Millipore system. The biogenic amine standards were purchased from Sigma-Aldrich.

LAB and yeast counts and implantation. Randomly amplified polymorphic DNA (RAPD)-based genotyping was used to determine the implantation of the $O$. oeni CECT 9749 strain as described below. Before inoculation with $O$. oeni CECT 9749, grape musts were checked to determine the amount of wild LAB from the vineyards (Figure 1A, red points). Then, wine samples were collected at three time points for LAB determination (at $O$. oeni inoculation, $48 \mathrm{hr}$ after inoculation, and in the second third of the MLF) (Figure 1A black points). Samples were serially diluted and seeded onto MLO plates (Zúñiga et al. 1993). Plates were counted, and 10 single colonies were randomly picked from plates with 30 to 300 colonies and suspended in $10 \mu \mathrm{L}$ of sterile milliU water (Millipore). These suspensions were used in RAPD-PCR amplification with M13 primers as described by Berbegal et al. (2017). In each electrophoresis gel, the electrophoretic band profiles obtained from LAB isolates and $O$. oeni CECT 9749 were compared to determine the percentage of implantation of the inoculated strain in each tank (Figure $1 \mathrm{C}$ and 1D).

A similar procedure was developed to check the cellular densities of yeasts and the implantation success of the inoculated yeast strain S. cerevisiae CECT 12008 (Figure 1B). Samples, taken at three stages of alcoholic fermentation (corresponding to densities $1.100,1.040$, and $0.990 \mathrm{~g} / \mathrm{cm}^{3}$ ), were seeded onto malt extract agar and incubated. Yeast colonies 

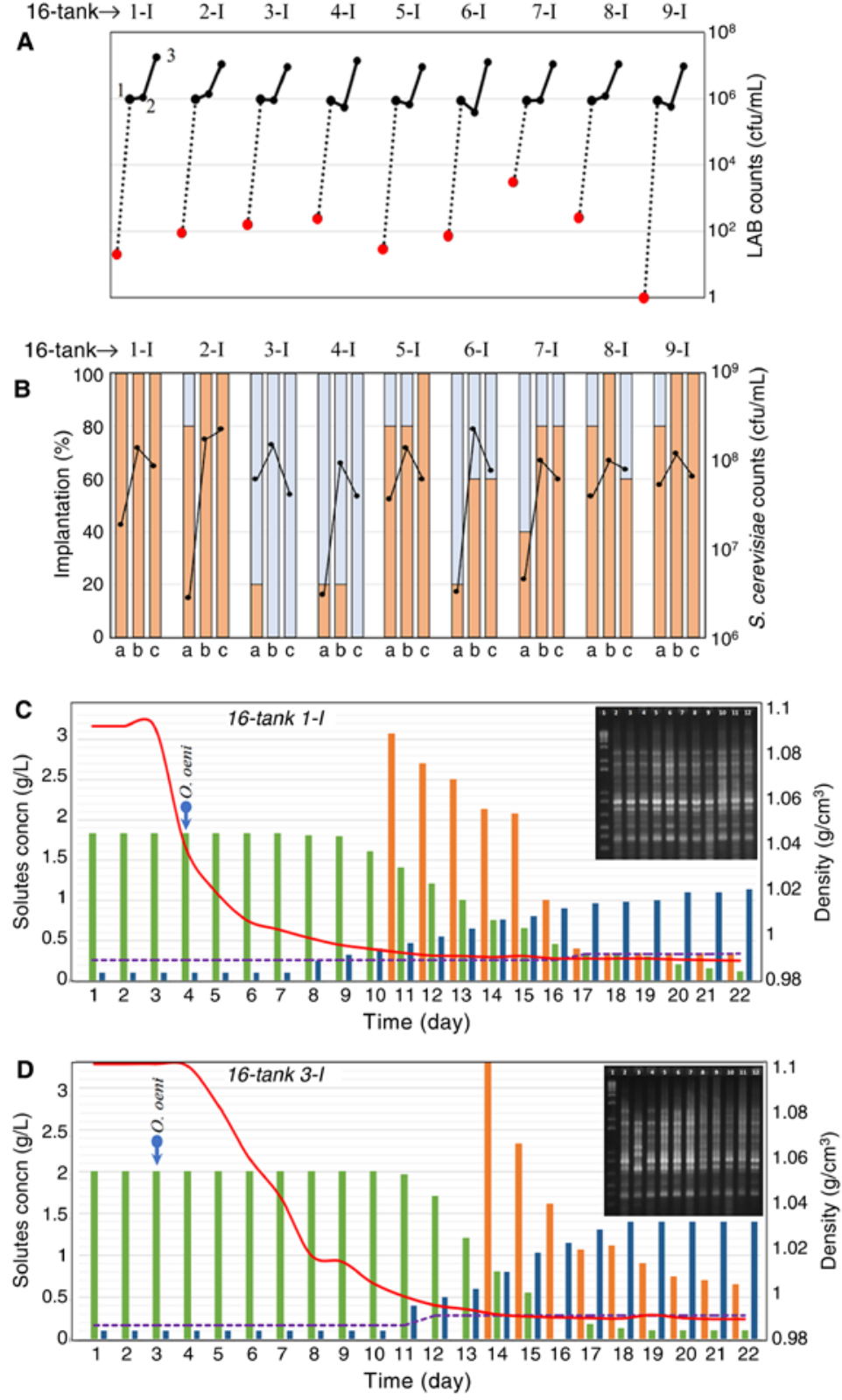

Figure 1 Microbiological control in nine tanks from the 2016 vintage (Table 2) to exemplify the evolution of the inoculated tanks with the strains Oenococcus oeni CECT 9749 and Saccharomyces cerevisiae CECT 12008. (A) lactic acid bacteria (LAB) cell counts. Three sampling times were used to follow $O$. oeni CECT 9749 development: 1, Inoculation of $O$. oeni; 2, LAB counts at $48 \mathrm{hr}$ after inoculation; and 3 , LAB counts after two-thirds of the malolactic fermentation (MLF). LAB counts before inoculation were also determined (red dots). (B) Progress of alcoholic fermentation (AF). $S$. cerevisiae cell counts and implantation of the selected yeast strain. Three samples were taken at different stages of AF to follow S. cerevisiae CECT 12008 development. Orange bars indicate the implantation percentage of the strain CECT 12008, whereas light blue bars indicate the implantation percentage of other wild $S$. cerevisiae strains. (C, D) Fermentation kinetics during AF and MLF in two tanks (16-Tank 1-I and 16-Tank 3-I) inoculated with O. oeni CECT 9749. Arrows indicate the moment of each inoculum addition. Malic acid (green), lactic acid (blue), glucose + fructose (orange), density (red), and acetic acid (violet). Glucose + fructose concentrations above $3 \mathrm{~g} / \mathrm{L}$ are not presented in order to facilitate the representation. Randomly amplified polymorphic DNA (RAPD)-fingerprinting images of $\mathrm{LAB}$ colonies isolated from each wine tank have been embedded at the upper-right of these figures. Lane 1: molecular weight markers; Lanes 2 to 11: RAPD band patterns of 10 randomly picked colonies; Lane 12: RAPD band pattern of $O$. oeni CECT 9749 . were counted, and 10 were randomly picked from the plates for genotyping. Colonies were suspended in water and subjected to PCR amplification of the interdelta regions (de Celis et al. 2019). The electrophoretic band pattern obtained for S. cerevisiae CECT 12008 was used for reliable comparisons within the same electrophoresis gel.

Analysis of BAs. Nine BAs (histamine, tyramine, putrescine, cadaverine, phenylethylamine, spermidine, agmatine, tryptamine, and isoamylamine) were analyzed using the method described by Gómez-Alonso et al. (2007) with slight modifications (Ortega-Heras et al. 2014). Aminoenone derivatives of amines were obtained by reaction with DEEMM and then analyzed by reverse-phase high performance liquid chromatography in an Agilent Technologies LC series 1200 with a diode-array detection system (Agilent).

Samples were taken after MLF and after 12 months of aging in barrels after their respective racking. BA analyses were carried out in duplicate.

Analysis of enological parameters. Standard enological parameters in wines were determined according to the official analysis methods of OIV: $\mathrm{pH}$, titratable acidity (as $\mathrm{g} / \mathrm{L}$ tartaric acid), and alcohol content (\%vol: $\mathrm{mL}$ ethanol/100 $\mathrm{mL}$ wine). Malic, lactic, and acetic acids were analyzed using enzymatic kits in a Y15 Analyser (Biosystems).

$\mathrm{SO}_{2}$ level was determined by the official analysis methods of OIV (OIV-MA-AS323-04A).

These enological parameters were analyzed in samples after AF and when MLF was finalized. Analyses were performed immediately after sample collection.

Statistical analyses. A one-way analysis of variance and a Fisher least significant difference test at a significance level of $p<0.05$ was performed using Statgraphics Centurion XVII software to determine the effects of inoculation and of aging in oak barrels on the BA content.

\section{Results}

O. oeni implantation. The selected $O$. oeni strain was implanted in all the inoculated wines and in almost all the non-inoculated ones. In eight non-inoculated wines from the 2016 vintage (tanks $15-\mathrm{S}$ to $22-\mathrm{S}$ ), the selected $O$. oeni strain was either not found or was found at levels under $50 \%$ (Table 2). In the 2017 and 2018 vintages, the inoculated strain was present in the whole winery. The LAB implantation percentage is shown in Tables 2 to 4 .

Figure $1 \mathrm{C}$ and $1 \mathrm{D}$ show the electrophoretic patterns (EP) of bands for 10 randomly picked colonies from MLO plates seeded with wines from two tanks of the 2016 vintage to determine the percentage of $O$. oeni implantation. Overall, a strain is considered to be implanted when it is clearly dominant, with a percentage of implantation higher than $80 \%$ (i.e., eight of $10 \mathrm{EP}$ are the same as the EP of the inoculated strain). In tank 16 1-I, the EP of all samples (lanes 2 to 11) were identical to the known EP (lane 12) of the inoculated O. oeni CECT 9749 strain; therefore, the implantation was considered complete. However, in tank 16 3-I, the EP of one sample did not match the EP of the inoculated $O$. oeni strain; thus, the implantation percentage was considered to be $\sim 90 \%$. 
Fermentation kinetics. Kinetics of malic acid consumption and lactic acid production followed the same pattern in all fermentations performed in the three harvests of the study. As an example, Figure 1C and 1D show that the malic acid and lactic acid kinetics during the progression of the fermentation were similar in two selected tanks (Tank 16 1-I and 16 3-I), one with good $(100 \%)$ implantation of the S. cerevisiae strain CECT 12008 (Tank 16 1-I, Figure 1B) and one (Tank $163-\mathrm{I}$, Figure 1B) with poor implantation $(0 \%$, similar to spontaneous). This fact, together with the results shown in Table 2, indicates minimal influence of the yeast on the progression of MLF.

BA analyses. Tables 2 to 4 show the BA concentrations found in all analyzed wines from the three vintages just after MLF and after 12 months of aging in barrels. Spermidine and phenylethylamine were found in low concentrations, and no statistically significant differences were observed between wines (data not shown). Agmatine, tryptamine, and isoamylamine were not detected in any wines. Therefore, the study focused on the content of the main BAs (histamine, tyramine, cadaverine, and putrescine) found in wines. Putrescine was the most abundant amine in all the wines, with concentrations ranging from 2.9 to $12.0 \mathrm{mg} / \mathrm{L}$.

A clear difference in histamine and putrescine content was observed between the inoculated and the non-inoculated wines from the 2016 vintage, both immediately after MLF and after 12 months of aging in barrels (Table 2). The noninoculated wines presented statistically significant higher histamine and putrescine concentrations than the inoculated ones. The wines implanted with the $O$. oeni strain showed reductions of $93.6 \%$ for histamine and $30.2 \%$ for putrescine. Furthermore, it should be noted that the non-inoculated wines in which the selected $O$. oeni strain was also implanted (tanks $10-\mathrm{S}$ to $14-\mathrm{S}$ ) showed the lowest histamine content.

In contrast, the same results were not observed in the wines from the 2017 and 2018 vintages (Tables 3 and 4). In these wines, inoculation did not produce statistically significant differences in histamine and putrescine concentrations. However, it should be taken into account that in these two vintages, the content of these BAs was very low, and the presence of the O. oeni CECT 9749 strain was detected in all the wines, both inoculated and non-inoculated.

Table 2 Biogenic amines of the wines from the 2016 vintage analyzed after malolactic fermentation (MLF) and after 12 months of aging in barrels. ${ }^{a} \mathrm{LAB}$, lactic acid bacteria.

\begin{tabular}{|c|c|c|c|c|c|c|c|c|c|c|c|}
\hline \multirow[b]{2}{*}{ Sample } & \multirow[b]{2}{*}{$\begin{array}{c}\text { Yeast } \\
\text { inoculation }\end{array}$} & \multirow[b]{2}{*}{$\begin{array}{c}\text { LAB } \\
\text { inoculation }\end{array}$} & \multirow{2}{*}{$\begin{array}{c}\text { LAB } \\
\text { implantation } \\
(\%)\end{array}$} & \multicolumn{2}{|c|}{ Histamine (mg/L) } & \multicolumn{2}{|c|}{ Tyramine (mg/L) } & \multicolumn{2}{|c|}{ Putrescine (mg/L) } & \multicolumn{2}{|c|}{ Cadaverine (mg/L) } \\
\hline & & & & $\begin{array}{l}\text { After } \\
\text { MLF }\end{array}$ & $\begin{array}{c}12 \\
\text { months }\end{array}$ & $\begin{array}{l}\text { After } \\
\text { MLF }\end{array}$ & $\begin{array}{c}12 \\
\text { months }\end{array}$ & $\begin{array}{l}\text { After } \\
\text { MLF }\end{array}$ & $\begin{array}{c}12 \\
\text { months }\end{array}$ & $\begin{array}{l}\text { After } \\
\text { MLF }\end{array}$ & $\begin{array}{c}12 \\
\text { months }\end{array}$ \\
\hline 16-Tank 1-I & yes & yes & 100 & $n d^{c}$ & 2.00 & 1.85 & 1.38 & 3.10 & 4.03 & 0.35 & 0.43 \\
\hline 16-Tank 2-I & yes & yes & 100 & nd & 1.39 & 1.54 & 1.36 & 3.42 & 2.99 & 0.41 & 0.33 \\
\hline 16-Tank 3-I & yes & yes & 90 & nd & 2.40 & 1.60 & 1.45 & 2.75 & 2.92 & 0.38 & 0.37 \\
\hline 16-Tank 4-I & yes & yes & 80 & 1.14 & 1.62 & 1.42 & 1.20 & 4.31 & 3.79 & 0.37 & 0.33 \\
\hline 16-Tank 5-I & yes & yes & 100 & nd & 1.18 & 1.29 & 1.35 & 2.36 & 2.43 & 0.34 & 0.32 \\
\hline 16-Tank 6-I & yes & yes & 100 & nd & 3.80 & 1.55 & 1.54 & 4.64 & 4.64 & 0.41 & 0.39 \\
\hline 16-Tank 7-I & yes & yes & 100 & nd & 1.00 & 1.51 & 1.31 & 3.72 & 3.43 & 0.37 & 0.36 \\
\hline 16-Tank ו-I & yes & yes & 100 & 1.11 & 1.76 & 1.54 & 1.33 & 3.42 & 3.24 & 0.35 & 0.34 \\
\hline 16-Tank 9-I & yes & yes & 100 & 1.16 & 1.41 & 2.20 & 1.69 & 2.99 & 2.91 & 0.42 & 0.38 \\
\hline \multicolumn{2}{|c|}{$\begin{array}{l}\text { MEAN } \\
\text { Standard deviation }\end{array}$} & & & $\begin{array}{c}0.38 \mathrm{a}, \mathrm{y} \\
0.57\end{array}$ & $\begin{array}{c}1.84 \mathrm{a}, \mathrm{z} \\
0.85\end{array}$ & $\begin{array}{c}1.61 \mathrm{a}, \mathrm{y} \\
0.27\end{array}$ & $\begin{array}{c}1.40 \mathrm{a}, \mathrm{y} \\
0.14\end{array}$ & $\begin{array}{c}3.41 \mathrm{a}, \mathrm{y} \\
0.73\end{array}$ & $\begin{array}{c}3.38 \mathrm{a}, \mathrm{y} \\
0.68\end{array}$ & $\begin{array}{c}0.38 \mathrm{a}, \mathrm{y} \\
0.03\end{array}$ & $\begin{array}{c}0.36 \mathrm{a}, \mathrm{y} \\
0.04\end{array}$ \\
\hline $16-$ Tank $10-S^{b}$ & yes & no & 100 & 2.64 & 6.88 & 2.22 & 1.91 & 4.10 & 42.5 & 0.50 & 0.44 \\
\hline 16-Tank 11-S & yes & no & 80 & 4.32 & 5.95 & 1.32 & 1.13 & 7.69 & 7.15 & 0.39 & 0.33 \\
\hline 16-Tank 12-S & yes & no & 100 & nd & 2.63 & 2.74 & 2.15 & 5.91 & 19.0 & 0.45 & 0.41 \\
\hline 16-Tank 13-S & no & no & 100 & nd & 3.54 & 1.54 & 1.45 & 3.58 & 3.31 & 0.39 & 0.35 \\
\hline 16-Tank 14-S & yes & no & 100 & 1.50 & 1.70 & 1.00 & 1.05 & 5.46 & 4.39 & 0.29 & 0.28 \\
\hline 16-Tank 15-S & yes & no & 0 & 16.4 & 16.3 & 1.87 & 1.84 & 10.1 & 12.0 & 0.53 & 0.47 \\
\hline 16-Tank 16-S & yes & no & 0 & 18.7 & 17.3 & 1.57 & 1.36 & 6.42 & 11.2 & 0.37 & 0.36 \\
\hline 16-Tank 17-S & yes & no & 0 & 12.8 & 15.8 & 1.73 & 1.45 & 4.40 & 4.55 & 0.35 & 0.32 \\
\hline 16-Tank 18-S & yes & no & 40 & 14.3 & 12.8 & 1.28 & 0.98 & 3.12 & 2.93 & 0.36 & 0.32 \\
\hline 16-Tank 19-S & yes & no & 0 & 15.7 & 17.4 & 1.66 & 1.42 & 3.87 & 17.6 & 0.36 & 0.41 \\
\hline 16-Tank 20-S & yes & no & 0 & 17.1 & 17.4 & 1.89 & 1.66 & 3.01 & 3.30 & 0.39 & 0.39 \\
\hline 16-Tank 21-S & yes & no & 0 & 8.45 & 12.6 & 1.61 & 5.94 & 4.78 & 24.8 & 0.28 & 0.81 \\
\hline 16-Tank 22-S & yes & no & 0 & 10.7 & 12.5 & 1.06 & 1.24 & 4.23 & 3.84 & 0.32 & 0.31 \\
\hline $\begin{array}{l}\text { MEAN } \\
\text { Standard deviat }\end{array}$ & & & & $\begin{array}{c}9.42 \mathrm{~b}, \mathrm{y} \\
6.96\end{array}$ & $\begin{array}{c}11.0 \mathrm{~b}, \mathrm{y} \\
6.02\end{array}$ & $\begin{array}{c}1.65 \mathrm{a}, \mathrm{y} \\
0.47\end{array}$ & $\begin{array}{c}1.81 \mathrm{a}, \mathrm{y} \\
1.29\end{array}$ & $\begin{array}{c}5.13 \mathrm{~b}, \mathrm{y} \\
2.01\end{array}$ & $\begin{array}{c}12.0 \mathrm{~b}, \mathrm{z} \\
11.59\end{array}$ & $\begin{array}{c}0.38 \mathrm{a}, \mathrm{y} \\
0.07\end{array}$ & $\begin{array}{c}0.40 \mathrm{a}, \mathrm{y} \\
0.14\end{array}$ \\
\hline
\end{tabular}

aMean values with different letters in each compound indicate statistically significant differences at $p<0.05$. Letters (a,b) indicate differences between the inoculated and the non-inoculated wines in the same aging stage and letters $(y, z)$ indicate differences between aging stages. Histamine values $>10 \mathrm{mg} / \mathrm{L}$ (above the recommendation by the International Organisation of Vine and Wine) are marked in bold.

bl: inoculated wines; S: spontaneous MLF or non-inoculated wines.

'nd: not detected. 
No statistically significant differences were found in the tyramine and cadaverine concentrations of the wines due to O. oeni inoculation, with the exception of wines from the 2017 vintage, in which small differences were found. After MLF, the inoculated 2017 wines had $1.30 \mathrm{mg} / \mathrm{L}$ of tyramine and $0.36 \mathrm{mg} / \mathrm{L}$ of cadaverine, while the non-inoculated wines had $1.70 \mathrm{mg} / \mathrm{L}$ of tyramine and $0.49 \mathrm{mg} / \mathrm{L}$ of cadaverine (Table 3).

After 12 months of aging in barrels, significant increases were recorded for putrescine in the non-inoculated wines from the 2016 vintage. Increases in BA content of the 2017 wines were observed, although in general, they were very low.

General enological characteristics. Mean values and standard deviations for the enological parameters of wines from the three consecutive vintages (2016, 2017, and 2018) at the end of AF and MLF are shown in Table 5. The wines from 2017 and 2018 showed a slightly higher alcohol content $(15.4 \% \mathrm{vol})$ than the 2016 wines $(14.8 \% \mathrm{vol})$. In addition, the 2017 wines showed slightly lower titratable acidity $(5.2 \mathrm{~g} / \mathrm{L})$ and malic acid content $(1.45 \mathrm{~g} / \mathrm{L})$ than the wines obtained from the 2016 and 2018 vintages (mean values of $5.9 \mathrm{~g} / \mathrm{L}$ tartaric acid and $1.9 \mathrm{~g} / \mathrm{L}$ malic acid). In spite of these slight differences, wines from the three vintages showed similar enological characteristics, with $\mathrm{pH}$ ranges $\sim 3.6$ to 3.8 , titratable acidities between 5.2 and $6.1 \mathrm{~g} / \mathrm{L}$ of tartaric acid, and alcoholic content between 14.7 and $15.6 \%$ vol.
Concerning the effect of the $O$. oeni inoculation, no statistically significant differences were found in most of the enological parameters between the inoculated and the noninoculated wines in each vintage, with the exception of the lactic and acetic acid concentrations in the 2018 wines.

All wines completed the MLF; malic acid concentrations were below $0.2 \mathrm{~g} / \mathrm{L}$. Before aging, the wines were corrected with $\mathrm{SO}_{2}$, and molecular $\mathrm{SO}_{2}$ was above 0.5 (Table 5), allowing it to maintain its antibacterial activity.

\section{Discussion}

O. oeni implantation. To ensure $O$. oeni implantation and avoid development of wild histaminogenic LAB, we required efficient growth of the selected $O$. oeni strain. Highly active cultures showing good malic acid enzymatic activity prevent sugar consumption, avoiding accumulation of acetic and lactic acids.

Several studies have reported that the inoculation of bacterial starters before or during AF (simultaneous inoculation of yeast and LAB) allows better control of MLF in winemaking (Massera et al. 2009, Azzolini et al. 2010, Smit and du Toit 2013, Izquierdo Cañas et al. 2015). In some preliminary experiments on this topic (data not shown) during the 2013, 2014, and 2015 harvests, we observed that when wines were inoculated with O. oeni CECT 9749 at the end of AF, wild

Table 3 Biogenic amines of the wines from the 2017 vintage analyzed after malolactic fermentation (MLF) and after 12 months of aging in barrels. ${ }^{a} \mathrm{LAB}$, lactic acid bacteria.

\begin{tabular}{|c|c|c|c|c|c|c|c|c|c|c|c|}
\hline \multirow[b]{2}{*}{ Sample } & \multirow[b]{2}{*}{$\begin{array}{c}\text { Yeast } \\
\text { inoculation }\end{array}$} & \multirow[b]{2}{*}{$\begin{array}{c}\text { LAB } \\
\text { inoculation }\end{array}$} & \multirow{2}{*}{$\begin{array}{c}\text { LAB } \\
\text { implantation } \\
(\%)\end{array}$} & \multicolumn{2}{|c|}{ Histamine (mg/L) } & \multicolumn{2}{|c|}{ Tyramine $(\mathrm{mg} / \mathrm{L})$} & \multicolumn{2}{|c|}{ Putrescine (mg/L) } & \multicolumn{2}{|c|}{ Cadaverine (mg/L) } \\
\hline & & & & $\begin{array}{l}\text { After } \\
\text { MLF }\end{array}$ & $\begin{array}{c}12 \\
\text { months }\end{array}$ & $\begin{array}{l}\text { After } \\
\text { MLF }\end{array}$ & $\begin{array}{c}12 \\
\text { months }\end{array}$ & $\begin{array}{l}\text { After } \\
\text { MLF }\end{array}$ & $\begin{array}{c}12 \\
\text { months }\end{array}$ & $\begin{array}{l}\text { After } \\
\text { MLF }\end{array}$ & $\begin{array}{c}12 \\
\text { months }\end{array}$ \\
\hline 17-Tank 1-I & yes & yes & 100 & $<\mathrm{LQ}^{\mathrm{c}}$ & 0.28 & 1.15 & 1.42 & 2.96 & 3.46 & 0.31 & 0.35 \\
\hline 17-Tank 2-I & yes & yes & 100 & 0.32 & 1.42 & 0.68 & 1.08 & 2.57 & 3.31 & 0.35 & 0.40 \\
\hline 17-Tank 3-I & yes & yes & 100 & $<\mathrm{LQ}$ & 0.41 & 1.60 & 1.98 & 2.87 & 3.54 & 0.37 & 0.41 \\
\hline 17-Tank ו-I & yes & yes & 100 & $<L Q$ & 0.35 & 1.22 & 1.57 & 2.60 & 3.11 & 0.36 & 0.41 \\
\hline 17-Tank 5-I & yes & yes & 100 & $\mathrm{nd}^{\mathrm{c}}$ & $<\mathrm{LQ}$ & 1.23 & 1.78 & 2.77 & 3.25 & 0.26 & 0.31 \\
\hline 17-Tank 6-I & yes & yes & 100 & nd & 0.58 & 2.03 & 2.87 & 2.95 & 3.58 & 0.39 & 0.47 \\
\hline 17-Tank 7-I & yes & yes & 100 & $<L Q$ & 0.61 & 1.70 & 1.91 & 2.68 & 3.46 & 0.46 & 0.53 \\
\hline 17-Tank 8-I & yes & yes & 80 & nd & nd & 1.19 & 1.53 & 2.91 & 3.52 & 0.39 & 0.45 \\
\hline 17-Tank 9-I & yes & yes & 100 & nd & 0.27 & 0.85 & 1.14 & 2.86 & 3.43 & 0.36 & 0.40 \\
\hline 17-Tank 10-I & yes & yes & 100 & nd & 0.39 & 1.31 & 1.62 & 3.70 & 4.45 & 0.34 & 0.39 \\
\hline \multicolumn{2}{|c|}{$\begin{array}{l}\text { MEAN } \\
\text { Standard deviation }\end{array}$} & & & $<\mathrm{LQ}$ a, y & $\begin{array}{c}0.43 \mathrm{a}, \mathrm{z} \\
0.40\end{array}$ & $\begin{array}{c}1.30 \mathrm{a}, \mathrm{y} \\
0.39\end{array}$ & $\begin{array}{c}1.69 \mathrm{a}, \mathrm{z} \\
0.51\end{array}$ & $\begin{array}{c}2.89 \mathrm{a}, \mathrm{y} \\
0.32\end{array}$ & $\begin{array}{c}3.51 \mathrm{a}, \mathrm{z} \\
0.36\end{array}$ & $\begin{array}{c}0.36 \mathrm{a}, \mathrm{y} \\
0.05\end{array}$ & $\begin{array}{c}0.41 \mathrm{a}, \mathrm{z} \\
0.06\end{array}$ \\
\hline 17-Tank 11-S & yes & no & 100 & $<\mathrm{LQ}$ & 0.51 & 1.62 & 1.87 & 2.48 & 3.24 & 0.47 & 0.53 \\
\hline 17-Tank 12-S & yes & no & 100 & nd & 0.28 & 1.91 & 2.49 & 2.95 & 4.80 & 0.48 & 0.58 \\
\hline 17-Tank 13-S & yes & no & 100 & $<\mathrm{LQ}$ & $<L Q$ & 2.00 & 2.17 & 2.80 & 3.74 & 0.54 & 0.56 \\
\hline 17-Tank 14-S & yes & no & 100 & nd & 0.36 & 1.91 & 2.18 & 3.02 & 3.81 & 0.47 & 0.53 \\
\hline 17-Tank 15-S & yes & no & 100 & $<\mathrm{LQ}$ & 0.43 & 1.59 & 1.78 & 2.34 & 3.31 & 0.44 & 0.50 \\
\hline 17-Tank 16-S & yes & no & 100 & 0.44 & 0.45 & 1.63 & 1.95 & 3.19 & 3.82 & 0.59 & 0.61 \\
\hline 17-Tank 17-S & no & no & 80 & 0.38 & 0.35 & 1.22 & 1.55 & 3.09 & 3.74 & 0.45 & 0.50 \\
\hline 17-Tank 18-S & no & no & 100 & nd & $<\mathrm{LQ}$ & 1.75 & 2.26 & 3.59 & 5.13 & 0.46 & 0.52 \\
\hline \multirow{2}{*}{\multicolumn{2}{|c|}{$\begin{array}{l}\text { MEAN } \\
\text { Standard deviation }\end{array}$}} & & & $<\mathrm{LQ}$ a, $\mathrm{y}$ & $0.80 \mathrm{a}, \mathrm{z}$ & $1.70 \mathrm{~b}, \mathrm{y}$ & $2.03 \mathrm{~b}, \mathrm{z}$ & $2.93 \mathrm{a}, \mathrm{y}$ & $3.95 b, z$ & $0.49 b, y$ & $0.54 \mathrm{~b}, \mathrm{z}$ \\
\hline & & & & & 0.43 & 0.25 & 0.30 & 0.40 & 0.67 & 0.05 & 0.04 \\
\hline
\end{tabular}

a Mean values with different letters in each compound indicate statistically significant differences at $p<0.05$. Letters (a,b) indicate differences between the inoculated and the non-inoculated wines in the same aging stage and letters $(y, z)$ indicate differences between aging stages. b: inoculated wines; S: spontaneous MLF or non-inoculated wines.

'LQ: limit of quantification $(0.20 \mathrm{mg} / \mathrm{L})$; nd: not detected. 
LAB counts from grapes were high, making it impossible to ensure the implantation of the inoculated strain. Consequently, histamine production was not reduced.

Therefore, in this study, it was decided to inoculate a selected non-histaminogenic $O$. oeni strain at the beginning of AF.

The proliferation of this strain was successful in all the inoculated wines of the three vintages. In the 2016 vintage, the selected $O$. oeni strain was found in $38 \%$ of the non-inoculated wines; in the 2017 and 2018 vintages, it was found in all the wines, independent of inoculation. This is a very interesting result because it has been observed that over the years, the selected autochthonous $O$. oeni strain became predominant in the production of wines in the whole winery, even those wines that were not previously inoculated. Therefore, the addition of the well-adapted culture of $O$. oeni CECT 9749 to the different tanks during AF seemed to generate a high prevalence of the strain in the three consecutive vintages studied. Similar results have been obtained using selected $S$. cerevisiae strains as starters for wine fermentation, indicating that these practices could have an important effect on microbial diversity in surrounding vineyards (de Celis et al. 2019).

In real winery conditions, yeast and bacteria do not work separately. Inoculated and non-inoculated tanks are processed using the same equipment (pumps, tubes, etc.), and because the inoculated tanks are routinely the first to be used for fermentation, the dissemination of the bacterial cultures to the non-inoculated tanks is possible.

BA analyses. The implantation results are in accordance with the BA data of these wines. The non-inoculated and nonLAB-implanted wines from 2016 showed the highest histamine content (Table 3). Histamine formation occurs mainly during MLF, and no significant increases were observed during aging in barrels, with some exceptions. A histamine increase was observed in the wines with very low initial concentrations,

Table 4 Biogenic amines of the wines from the 2018 vintage analyzed after malolactic fermentation (MLF) and after 12 months of aging in barrels. ${ }^{a} \mathrm{LAB}$, lactic acid bacteria.

\begin{tabular}{|c|c|c|c|c|c|c|c|c|c|c|c|}
\hline \multirow[b]{2}{*}{ Sample } & \multirow[b]{2}{*}{$\begin{array}{c}\text { Yeast } \\
\text { inoculation }\end{array}$} & \multirow[b]{2}{*}{$\begin{array}{c}\text { LAB } \\
\text { inoculation }\end{array}$} & \multirow{2}{*}{$\begin{array}{c}\text { LAB } \\
\text { implantation } \\
(\%)\end{array}$} & \multicolumn{2}{|c|}{ Histamine (mg/L) } & \multicolumn{2}{|c|}{ Tyramine (mg/L) } & \multicolumn{2}{|c|}{ Putrescine (mg/L) } & \multicolumn{2}{|c|}{ Cadaverine (mg/L) } \\
\hline & & & & $\begin{array}{l}\text { After } \\
\text { MLF }\end{array}$ & $\begin{array}{c}12 \\
\text { months }\end{array}$ & $\begin{array}{l}\text { After } \\
\text { MLF }\end{array}$ & $\begin{array}{c}12 \\
\text { months }\end{array}$ & $\begin{array}{l}\text { After } \\
\text { MLF }\end{array}$ & $\begin{array}{c}12 \\
\text { months }\end{array}$ & $\begin{array}{l}\text { After } \\
\text { MLF }\end{array}$ & $\begin{array}{c}12 \\
\text { months }\end{array}$ \\
\hline 18-Tank 1-I & yes & yes & 100 & $<L^{c}$ & $<L Q$ & 1.53 & 1.50 & 3.61 & 3.47 & 0.45 & 0.42 \\
\hline 18-Tank 2-I & yes & yes & 100 & 0.39 & 0.34 & 1.23 & 1.53 & 2.10 & 2.33 & 0.44 & 0.42 \\
\hline 18-Tank ו-I & yes & yes & 100 & 0.42 & 0.40 & 2.31 & 2.32 & 4.49 & 4.26 & 0.56 & 0.52 \\
\hline 18-Tank ו-I & yes & yes & 100 & 0.41 & 0.38 & 1.88 & 1.65 & 5.04 & 4.65 & 0.55 & 0.48 \\
\hline 18-Tank ו-I & yes & yes & 100 & 0.32 & 0.30 & 2.13 & 2.30 & 3.03 & 2.98 & 0.52 & 0.49 \\
\hline 18-Tank 6-I & yes & yes & 100 & 0.62 & 0.59 & 2.49 & 2.27 & 3.35 & 3.13 & 0.59 & 0.53 \\
\hline 18-Tank 7-I & yes & yes & 100 & 0.62 & 0.57 & 2.58 & 2.35 & 3.31 & 3.10 & 0.59 & 0.53 \\
\hline 18-Tank 8-I & yes & yes & 100 & 0.45 & 0.42 & 1.54 & 1.57 & 3.21 & 3.02 & 0.45 & 0.41 \\
\hline 18-Tank 9-I & yes & yes & 100 & 0.50 & 0.51 & 1.56 & 1.61 & 3.21 & 3.18 & 0.44 & 0.42 \\
\hline 18-Tank 10-I & yes & yes & 100 & 0.41 & 0.51 & 2.58 & 1.85 & 4.63 & 3.22 & 0.47 & 0.57 \\
\hline 18-Tank 11-I & yes & yes & 100 & 0.38 & 0.40 & 2.31 & 2.26 & 4.54 & 4.31 & 0.57 & 0.51 \\
\hline 18-Tank 12-I & yes & yes & 100 & 0.42 & 0.41 & 2.15 & 2.17 & 3.74 & 3.62 & 0.56 & 0.51 \\
\hline 18-Tank 13-I & yes & yes & 100 & 0.46 & 0.45 & 2.49 & 2.41 & 3.93 & 3.76 & 0.58 & 0.54 \\
\hline 18-Tank 14-I & yes & yes & 100 & 0.31 & 0.30 & 2.93 & 2.92 & 4.35 & 4.65 & 0.56 & 0.64 \\
\hline 18-Tank 15-I & yes & yes & 100 & 0.58 & 0.60 & 2.65 & 2.44 & 3.46 & 3.29 & 0.59 & 0.50 \\
\hline 18-Tank 16-I & yes & yes & 100 & 0.26 & 0.28 & 3.30 & 3.15 & 4.04 & 3.87 & 0.67 & 0.61 \\
\hline \multicolumn{2}{|c|}{$\begin{array}{l}\text { MEAN } \\
\text { Standard deviation }\end{array}$} & & & $\begin{array}{c}0.42 \mathrm{a}, \mathrm{y} \\
0.12\end{array}$ & $\begin{array}{c}0.42 \mathrm{a}, \mathrm{y} \\
0.12\end{array}$ & $\begin{array}{c}2.23 \mathrm{a}, \mathrm{y} \\
0.56\end{array}$ & $\begin{array}{c}2.14 \mathrm{a}, \mathrm{y} \\
0.49\end{array}$ & $\begin{array}{c}3.75 \mathrm{a}, \mathrm{y} \\
0.75\end{array}$ & $\begin{array}{c}3.55 \mathrm{a}, \mathrm{y} \\
0.66\end{array}$ & $\begin{array}{c}0.54 \mathrm{a}, \mathrm{y} \\
0.07\end{array}$ & $\begin{array}{c}0.51 \mathrm{a}, \mathrm{y} \\
0.07\end{array}$ \\
\hline $18-$ Tank $17-S^{b}$ & yes & no & 100 & 0.32 & 0.40 & 1.52 & 1.54 & 3.01 & 3.12 & 0.45 & 0.47 \\
\hline 18-Tank 18-S & no & no & 100 & 0.28 & 0.88 & 1.79 & 2.44 & 4.55 & 5.03 & 0.41 & 0.46 \\
\hline 18-Tank 19-S & yes & no & 100 & 0.50 & 0.53 & 1.63 & 1.59 & 3.25 & 3.25 & 0.51 & 0.46 \\
\hline 18-Tank 20-S & no & no & 100 & 0.46 & 0.74 & 1.83 & 1.94 & 4.60 & 4.27 & 0.50 & 0.45 \\
\hline 18-Tank 21-S & no & no & 100 & 0.45 & 0.72 & 2.05 & 2.00 & 4.39 & 4.30 & 0.48 & 0.47 \\
\hline 18-Tank 22-S & no & no & 100 & 0.56 & 0.94 & 2.19 & 2.16 & 4.52 & 4.12 & 0.49 & 0.45 \\
\hline 18-Tank 23-S & yes & no & 100 & 0.41 & 0.75 & 2.58 & 2.55 & 4.63 & 4.50 & 0.47 & 0.45 \\
\hline 18-Tank 24-S & no & no & 100 & 0.50 & 0.61 & 2.70 & 2.74 & 4.71 & 4.37 & 0.40 & 0.42 \\
\hline 18-Tank 25-S & yes & no & 100 & 0.52 & 0.55 & 3.02 & 3.03 & 4.60 & 4.09 & 0.52 & 0.45 \\
\hline 18-Tank 26-S & no & no & 100 & 0.42 & 0.49 & 2.43 & 2.38 & 3.48 & 3.62 & 0.61 & 0.55 \\
\hline 18-Tank 27-S & yes & no & 100 & 0.26 & 0.44 & 2.11 & 1.86 & 4.30 & 3.96 & 0.56 & 0.48 \\
\hline \multirow{2}{*}{\multicolumn{2}{|c|}{$\begin{array}{l}\text { MEAN } \\
\text { Standard deviation }\end{array}$}} & & & $0.42 \mathrm{a}, \mathrm{y}$ & $0.64 b, z$ & $2.17 \mathrm{a}, \mathrm{y}$ & $2.20 \mathrm{a}, \mathrm{y}$ & $4.19 \mathrm{a}, \mathrm{y}$ & $4.06 \mathrm{~b}, \mathrm{y}$ & $0.49 \mathrm{a}, \mathrm{y}$ & $0.47 \mathrm{a}, \mathrm{y}$ \\
\hline & & & & 0.10 & 0.18 & 0.47 & 0.47 & 0.62 & 0.56 & 0.06 & 0.03 \\
\hline
\end{tabular}

aMean values with different letters in each compound indicate statistically significant differences at $p<0.05$. Letters (a, b) indicate differences between the inoculated and the non-inoculated wines in the same aging stage and letters $(y, z)$ indicate differences between aging stages. bl: inoculated wines; S: spontaneous MLF or non-inoculated wines.

'LQ: limit of quantification $(0.20 \mathrm{mg} / \mathrm{L})$. 
but this increase is not considered significant as the histamine levels were always less than $1 \mathrm{mg} / \mathrm{L}$. The results of previous studies are contradictory with respect to whether BA formation occurs mainly during MLF or during aging (Soufleros et al. 1998, Lonvaud-Funel 1999, Jiménez-Moreno et al. 2003, Landete et al. 2005, Marcobal et al. 2006, Hernández-Orte et al. 2008), and also are dependent on whether the MLF was carried out spontaneously or by inoculating LAB (Marcobal et al. 2006, Hernández-Orte et al. 2008). Hernández-Orte et al. (2008) showed an increase of histamine concentration after six months of oak aging that was higher in wines that conducted MLF with indigenous bacteria than in inoculated wines. Other authors indicated that LAB inoculation did not give rise to an increase of histamine during oak aging (Marcobal et al. 2006). The contradictory results found by different authors could be due to the prevalence, or lack thereof, of the non-histaminogenic strain, as some researchers evaluated the BA content but not the LAB strain that carried out the MLF. Our results indicate that the presence of the non-histamineproducing $O$. oeni strain during MLF prevents the increase of histamine values during aging.

Figure 2 shows the mean values of histamine in the inoculated and non-inoculated wines from the three vintages both immediately after MLF and after 12 months of aging in barrels. The use of the selected autochthonous $O$. oeni strain from the red wines of the winery drastically reduced the histamine content in the wines. A progressive reduction in histamine content in wines has been observed over the years. Mean histamine values decreased from $6 \mathrm{mg} / \mathrm{L}$ in inoculated wines and $18 \mathrm{mg} / \mathrm{L}$ in non-inoculated wines in 2011 (Berbegal et al. 2017) to $<1 \mathrm{mg} / \mathrm{L}$ in wines of the 2017 and 2018 vintages. These low histamine values were also found in the non-inoculated wines, but only in those where the selected $O$. oeni strain has been implanted. This might be due to the prevalence of the selected $O$. oeni strain against other indigenous LAB that manage to avoid the growth of other populations' histamine producers. In addition, this leads to the continuation of low histamine levels during aging in barrels. Therefore, it seems that the implantation of the selected $O$. oeni strain is effective at reducing the risk of histamine formation and obtaining wines with low BA concentrations. The influence of the selected $O$. oen $i$ strain on the histamine and putrescine content was more significant than the effect on the cadaverine and tyramine content.

Putrescine concentrations were also significantly higher in the non-inoculated wines than in the inoculated onesbut only in the wines from 2016. Therefore, the indigenous

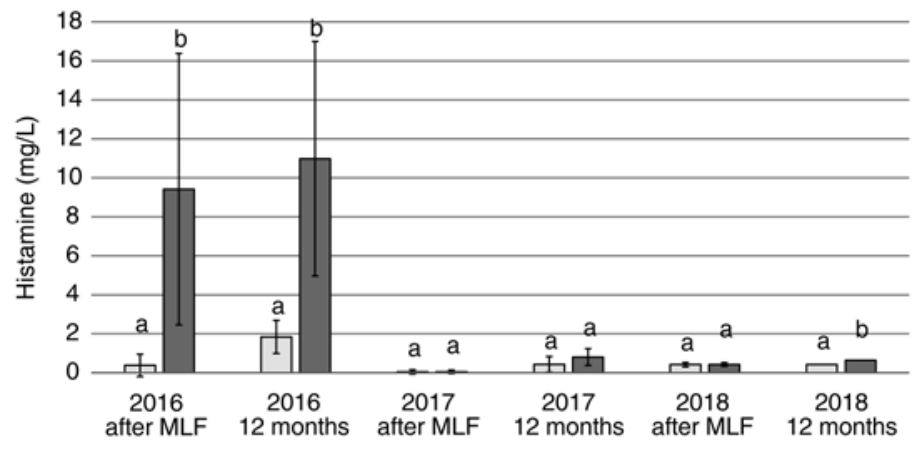

Figure 2 Mean values of histamine in the lactic acid bacteria-inoculated (light grey) and non-inoculated (dark grey) wines from the three vintages studied after malolactic fermentation (MLF) and after 12 months of aging in barrels. Mean values with different letters indicate statistically significant differences at $p<0.05$.

Table 5 Mean values and standard deviations of enological parameters of the inoculated and non-inoculated wines from the three vintages. ${ }^{a}$

\begin{tabular}{|c|c|c|c|c|c|c|c|c|c|c|}
\hline & & \multicolumn{4}{|c|}{ After alcoholic fermentation } & \multicolumn{3}{|c|}{ After malolactic fermentation } & \multicolumn{2}{|c|}{ Before aging } \\
\hline & & $\mathrm{pH}$ & $\begin{array}{c}\text { Titratable } \\
\text { acidity }^{\mathrm{b}}\end{array}$ & $\begin{array}{c}\text { Malic acid } \\
(\mathrm{g} / \mathrm{L})\end{array}$ & $\begin{array}{l}\text { Lactic acid } \\
(\mathrm{g} / \mathrm{L})\end{array}$ & $\begin{array}{l}\text { Lactic acid } \\
(\mathrm{g} / \mathrm{L})\end{array}$ & $\begin{array}{c}\text { Acetic acid } \\
(\mathrm{g} / \mathrm{L})\end{array}$ & Alcohol ${ }^{b}$ & $\begin{array}{c}\text { Free SO} \\
(\mathrm{mg} / \mathrm{L})\end{array}$ & $\begin{array}{c}\text { Molecular } \\
\mathrm{SO}_{2}\end{array}$ \\
\hline \multicolumn{11}{|l|}{2016 vintage } \\
\hline \multirow[t]{2}{*}{ Inoculated } & Mean & 3.71 & 6.02 & 1.92 & 0.28 & 1.26 & 0.40 & 14.8 & 52 & 0.80 \\
\hline & $\mathrm{SD}^{\mathrm{c}}$ & 0.10 & 0.40 & 0.16 & 0.09 & 0.14 & 0.04 & 0.5 & 3 & 0.11 \\
\hline \multirow[t]{2}{*}{ Non-inoculated } & Mean & 3.72 & 5.65 & 1.87 & 0.25 & 1.17 & 0.40 & 14.7 & 51 & 0.71 \\
\hline & SD & 0.07 & 0.47 & 0.19 & 0.15 & 0.11 & 0.04 & 0.4 & 4 & 0.10 \\
\hline \multicolumn{11}{|l|}{2017 vintage } \\
\hline \multirow[t]{2}{*}{ Inoculated } & Mean & 3.78 & 5.20 & 1.59 & 0.56 & 1.21 & 0.43 & 15.4 & 49 & 0.66 \\
\hline & SD & 0.07 & 0.42 & 0.59 & 0.16 & 0.10 & 0.06 & 0.4 & 4 & 0.09 \\
\hline \multirow[t]{2}{*}{ Non-inoculated } & Mean & 3.82 & 5.25 & 1.27 & 0.66 & 1.24 & 0.47 & 15.6 & 51 & 0.64 \\
\hline & SD & 0.11 & 0.31 & 0.46 & 0.34 & 0.13 & 0.09 & 0.2 & 4 & 0.11 \\
\hline \multicolumn{11}{|l|}{2018 vintage } \\
\hline \multirow[t]{2}{*}{ Inoculated } & Mean & 3.65 & 6.08 & 1.91 & $0.57 \mathrm{~b}$ & $1.60 \mathrm{~b}$ & $0.50 \mathrm{~b}$ & 15.4 & 49 & 0.56 \\
\hline & SD & 0.10 & 0.59 & 0.64 & 0.39 & 0.23 & 0.10 & 0.3 & 8 & 0.10 \\
\hline \multirow[t]{2}{*}{ Non-inoculated } & Mean & 3.59 & 5.99 & 2.05 & $0.24 \mathrm{a}$ & $1.31 \mathrm{a}$ & $0.42 \mathrm{a}$ & 15.4 & 47 & 0.57 \\
\hline & SD & 0.10 & 0.48 & 0.66 & 0.07 & 0.18 & 0.07 & 0.3 & 9 & 0.12 \\
\hline
\end{tabular}

${ }^{a}$ Mean values with different letters in each compound and vintage indicate statistically significant differences at $p<0.05$. Values without letters do not show statistically significant differences.

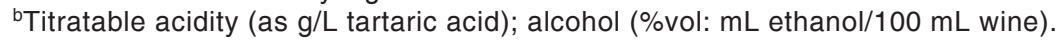

'SD: standard deviation. 
microbiota that conducted the MLF in these wines were responsible for producing these BAs. These results are in agreement with those found by other researchers who indicated that spontaneous MLF has a greater risk of producing wines with high BA content (Izquierdo Cañas et al. 2008, Berbegal et al. 2017).

Significant increases were recorded for putrescine in the non-inoculated wines during the aging process, mainly in the 2016 vintage. Putrescine can be formed by the decarboxylation of ornithine through the action of bacterial decarboxylase enzymes. Previously, Mangani et al. (2005) reported that $O$. oeni strains can produce putrescine not only from ornithine but also from arginine if they have the enzyme necessary to degrade arginine to ornithine. Arginine is one of the major amino acids found in grapes and wine and may be primarily responsible for the formation of putrescine. The increase observed in the mean putrescine values from 5.13 to $12.0 \mathrm{mg} / \mathrm{L}$ was mainly due to the large increases found in four wines (non-inoculated tanks $10-\mathrm{S}, 12-\mathrm{S}, 19-\mathrm{S}$, and $21-\mathrm{S}$ from the 2016 vintage). These wines also showed large decreases in arginine (between 40 and 77\%) and ornithine (between 36 and 94\%) during the aging time (data not shown). Therefore, although there was not a linear correlation between the degradation of the precursor amino acids and the formation of putrescine, the decrease in the concentrations of these amino acids can lead to the formation of this BA by indigenous bacteria that survived after MLF. Putrescine can reduce sensory quality at levels of 20 to $30 \mathrm{mg} / \mathrm{L}$ in red wines (Barbieri et al. 2019), but only the non-inoculated wine in tank 10-S from the 2016 vintage exceeded these values.

In general, the inoculation did not influence the content of tyramine and cadaverine. The non-inoculated wines from 2017 showed slightly higher levels of tyramine and cadaverine than the inoculated ones. However, these differences are not considered important because the values were low (similar to those found in the 2016 vintage and lower than those found in prior studies) (Marcobal et al. 2006, Hernández-Orte et al. 2008, Izquierdo Cañas et al. 2008, EFSA 2011). These BAs can be found in grapes before AF and MLF, and their amounts are related to the degree of grape maturation and the grape variety.

After 12 months of aging in barrels, no statistically significant differences were found in tyramine and cadaverine concentrations in the inoculated or non-inoculated wines. There was a slight increase for the 2017 wines, with mean final concentrations of less than $2.5 \mathrm{mg} / \mathrm{L}$ tyramine and 0.61 $\mathrm{mg} / \mathrm{L}$ cadaverine. These results are in accordance with those found by Marcobal et al. (2006) and Hernández-Orte et al. (2008), neither of whom found an increase of these BAs after six or 12 months of aging in barrels. The mean values of these BAs in our wines were lower than those reported by other authors (Marcobal et al. 2006, Hernández-Orte et al. 2008, Izquierdo Cañas et al. 2008, EFSA 2011) and similar to the concentrations reported by the European Food Safety Authority (EFSA) (2011).

Although the toxicological role of BAs in wines is still not well known, winemakers desire to avoid their formation and obtain wines with low BA concentrations, allowing the production of healthier wines that are less likely to cause allergic reactions (Ancín-Azpilicueta et al. 2019). The levels of histamine found in the wines of this study were lower than those reported by the EFSA (2011), with the exception of the wines in which the selected $O$. oeni strain was not implanted. In addition, the concentrations of tyramine, putrescine, and cadaverine in all the studied wines were within the range of data reported by the EFSA (2011).

General enological characteristics. AF was generally conducted by the inoculation of a selected $S$. cerevisiae CECT 12008 strain (Tables 2 to 4), with no influence of the yeast strain on the compounds and parameters analyzed.

The LAB inoculation also did not produce changes in the enological parameters of the wines (Table 5). The only statistically significant differences between the inoculated wines and the non-inoculated wines were found in the lactic and acetic acid concentrations of the 2018 wines. However, these differences in acetic acid were very small, with mean values ranging from 0.42 to $0.50 \mathrm{~g} / \mathrm{L}$.

In contrast, Mendoza et al. (2011) showed an increase in volatile acidity in co-inoculated musts due to bacterial sugar catabolism. This reaction is more favorable in wines with high $\mathrm{pH}$ values. In this study, only the inoculated wines from the 2018 vintage showed a slightly higher acetic acid concentration than the non-inoculated ones. However, the acetic acid values of $0.5 \mathrm{~g} / \mathrm{L}$ do not cause any negative effect in wines. Acclimatization of the culture in a medium rich in malic acid activates the malolactic metabolism of the culture and avoids bacterial sugar catabolism during AF. Therefore, the selected $O$. oeni strain did not produce an increase in volatile acidity, even when inoculated during AF, which agrees with the results obtained by other authors (Massera et al. 2009, Azzolini et al. 2010, Izquierdo Cañas et al. 2015).

Alexandre et al. (2004) and Muñoz et al. (2014) observed that the use of simultaneous inoculation of yeasts and LAB resulted in sluggish AF. However, in this study, the inoculation of the selected $O$. oeni strain did not interfere with the yeast growth, and the AF showed adequate development in all the wines. Both the yeast and the LAB used in this study were selected from the autochthonous microbiota of the surrounding vineyards and did not give rise to the negative effects found by other authors, such as an increase in volatile acidity or sluggish AF, effects that can be strain dependent.

In spite of the high alcohol content of the wines $(\sim 15$ to $15.5 \% \mathrm{vol}$ ), the MLF developed adequately and was finalized in all the wines. Thus, the selected $O$. oeni strain was adapted gradually to the ethanol content and wine characteristics (Jussier et al. 2006, Zapparoli et al. 2009, Azzolini et al. 2010), resistant to high alcohol content ( $>14.5 \% \mathrm{vol})$, and able to compete with other bacterial species, such as Pediococcus or Lactobacillus, that could reach the wines from the vineyards (Lonvaud-Funel 2001, López et al. 2008, Berbegal et al. 2017).

Most of the research on the influence of the inoculation of LAB starters has been carried out at the laboratory or pilot scale (Zapparoli et al. 2009, Azzolini et al. 2010, Mendoza 
et al. 2011, Izquierdo Cañas et al. 2015, Muñoz et al. 2014, Ortega-Heras et al. 2014). Therefore, our results are particularly important as this study was conducted in real winemaking conditions $(18,000 \mathrm{~kg}$ of grapes in $25,000-\mathrm{L}$ tanks) with 67 wines in three consecutive vintages.

\section{Conclusions}

The selection of a non-histaminogenic O. oeni strain, together with adequate adaptation of the culture to wine conditions at the beginning of each harvest, is a good strategy to avoid the formation of BAs, primarily histamine, in red wines.

This practice ensures the prevalence of the selected and inoculated O. oeni CECT 9749 strain —which can be present even in the non-inoculated wines - against the indigenous microbiota and allows reduction in histamine content of all the wines of the winery.

The success of the use of the selected $O$. oeni strain in the wines to avoid the production of BAs, mainly histamine, was maintained after 12 months of aging in barrels.

This procedure prevents microbial alterations during the points of fermentation in which wines are not protected with $\mathrm{SO}_{2}$. For this reason, less sulfide needs to be added for wine protection, and this method can be a tool for making wines with very low or no sulfide content.

\section{Literature Cited}

Alexandre H, Costello PJ, Remize F, Guzzo J and Guilloux-Benatier M. 2004. Saccharomyces cerevisiae-Oenococcus oeni interaction in wine: current knowledge and perspectives. Int J Food Microbiol 93:141-154

Ancín-Azpilicueta C, González-Marco A and Jiménez-Moreno N. 2008. Current knowledge about the presence of amines in wine. Crit Rev Food Sci Nutr 48:257-275.

Ancín-Azpilicueta C, Jiménez-Moreno N and Sola-Larrañaga C. 2019. Wine. In Innovations in Traditional Foods. Galanakis CM (ed.), pp. 221-256. Woodhead Publishing, Elsevier Inc.

Azzolini M, Tosi E, Vagnoli P, Krieger S and Zapparoli G. 2010. Evaluation of technological effects of yeast-bacterial co-inoculation in red table wine production. Ital J Food Sci 22:257-263.

Barbieri F, Montanari C, Gardini F and Tabanelli G. 2019. Biogenic amine production by lactic acid bacteria: A review. Foods 8:17.

Benito S. 2019a. The management of compounds that influence human health in modern winemaking from an HACCP point of view. Fermentation 5:33.

Benito S. 2019b. The impacts of Schizosaccharomyces on winemaking. Appl Microbiol Biotechnol 103:4291-4312.

Berbegal C, Benavent-Gil Y, Pardo I and Ferrer S. 2015. A novel culture medium for Oenococcus oeni malolactic starter production. LWT-Food Sci Technol 64:25-31.

Berbegal C, Benavent-Gil Y, Navascués E, Calvo A, Albors C, Pardo I and Ferrer S. 2017. Lowering histamine formation in a red Ribera del Duero wine (Spain) by using an indigenous $O$. oeni strain as a malolactic starter. Int J Food Microbiol 244:11-18.

Costantini A, García-Moruno E and Moreno-Arribas MV. 2009. Biochemical transformations produced by malolactic fermentation. In Wine Chemistry and Biochemistry. Moreno-Arribas MV and Polo MC (eds.), pp. 27-57. Springer, New York. de Celis M, Ruiz J, Martín-Santamaría M, Alonso A, Marquina D, Navascués E, Gómez-Flechoso M, Belda I and Santos A. 2019. Diversity of Saccharomyces cerevisiae yeasts associated to spontaneous and inoculated fermenting grapes from Spanish vineyards. Lett Appl Microbiol 68:580-588.

Del Prete V, Costantini A, Cecchini F, Morassut M and García-Moruno E. 2009. Occurrence of biogenic amines in wine: The role of grapes. Food Chem 112:474-481.

European Food Safety Authority (EFSA). 2011. EFSA Panel on Biological Hazards (BIOHAZ). Scientific opinion on risk base control of biogenic amine formation in fermented foods. EFSA J 9:2393,

García-Villar N, Hernández-Cassou S and Saurina J. 2007. Characterization of wines through the biogenic amine contents using chromatographic techniques and chemometric data analysis. J Agric Food Chem 55:7453-7461.

Gardini F, Özogul Y, Suzzi G, Tabanelli G and Özogul F. 2016. Technological factors affecting biogenic amine content in foods: A review. Front Microbiol 7:1218.

Gómez-Alonso S, Hermosín-Gutiérrez I and García-Romero E. 2007. Simultaneous HPLC analysis of biogenic amines, amino acids and ammonium ion as aminoenone derivatives in wine and beer samples. J Agric Food Chem 55:608-613.

Hernández-Orte P et al. 2008. Biogenic amine determination in wine fermented in oak barrels: Factors affecting formation. Food Res Int 41:697-706.

Izquierdo Cañas PM, García Romero E, Gómez Alonso S, Fernández González M and Palop Herreros MLL. 2008. Amino acids and biogenic amines during spontaneous malolactic fermentation in Tempranillo red wines. J Food Comp Anal 21:731-735.

Izquierdo Cañas PM, Gómez Alonso S, Ruiz Pérez P, Seseña Prieto S, García Romero E and Palop Herreros MLL. 2009. Biogenic amine production by Oenococcus oeni isolates from malolactic fermentation of Tempranillo wine. J Food Prot 72:907-910.

Izquierdo Cañas PM, García Romero E, Pérez-Martín F, Seseña S and Llanos Palop M. 2015. Sequential inoculation versus co-inoculation in Cabernet Franc wine fermentation. Food Sci Technol Int 21:203-212.

Jiménez-Moreno N, Torrea Goñi D and Ancín-Azpilicueta C. 2003. Changes in amine concentrations during aging of red wine in oak barrels. J Agric Food Chem 51:5732-5737.

Jussier D, Dubé Morneau A and Mira de Orduña R. 2006. Effect of simultaneous inoculation with yeast and bacteria on fermentation kinetics and key wine parameters of cool-climate Chardonnay. Appl Environ Microbiol 72:221-227.

Ladero V, Calles-Enríquez M, Fernández M and Álvarez MA. 2010. Toxicological effects of dietary biogenic amines. Curr Nutr Food Sci 6:145-156.

Landete JM, Ferrer S, Polo L and Pardo I. 2005. Biogenic amines in wines from three Spanish regions. J Agric Food Chem 53:1119-1124.

Lonvaud-Funel A. 1999. Lactic acid bacteria in the quality improvement and depreciation of wine. Antonie Van Leeuwenhoek 676:317-331.

Lonvaud-Funel A. 2001. Biogenic amines in wines: Role of lactic acid bacteria. FEMS Microbiol Lett 199:9-13.

López I, López R, Santamaría P, Torres C and Ruiz-Larrea F. 2008. Performance of malolactic fermentation by inoculation of selected Lactobacillus plantarum and Oenococcus oeni strains isolated from Rioja red wines. Vitis 47:123-129.

Mangani S, Guerrini S, Granchi L and Vincenzini M. 2005. Putrescine accumulation in wine: Role of Oenococcus oeni. Curr Microbiol 51:6-10.

Marcobal A, Martín-Álvarez PJ, Polo MC, Muñoz R and MorenoArribas MV. 2006. Formation of biogenic amines throughout the industrial manufacture of red wine. J Food Prot 69:397-404. 
Martuscelli M, Arfelli G, Manetta AC and Suzzi G. 2013. Biogenic amines content as a measure of the quality of wines of Abruzzo (Italy). Food Chem 140:590-597.

Massera A, Soria A, Catania C, Krieger S and Combina M. 2009. Simultaneous inoculation of Malbec (Vitis vinifera) musts with yeast and bacteria: Effects on fermentation performance, sensory and sanitary attributes of wines. Food Technol Biotechnol 47:192-201.

Mendoza LM, Merín MG, Morata VI and Farías ME. 2011. Characterization of wines produced by mixed culture of autochthonous yeasts and Oenococcus oeni from the northwest region of Argentina. J Ind Microbiol Biotechnol 38:1777-1785.

Moreno-Arribas MV and Polo MC. 2009. Amino acids and biogenic amines. In Wine Chemistry and Biochemistry. Moreno-Arribas MV and Polo MC (eds.), pp. 163-189. Springer, New York.

Moreno-Arribas MV, Polo MC, Jorganes F and Muñoz R. 2003. Screening of biogenic amine production by lactic acid bacteria isolated from grape must and wine. Int J Food Microbiol 84:117-123.

Moreno-Arribas MV, Smit AY and du Toit M. 2010. Biogenic amines and the winemaking process. In Managing Wine Quality. Reynolds AG (ed.), pp. 494-522. Woodhead Publishing Limited, Cambridge, UK.

Muñoz V, Beccaria B and Abreo E. 2014. Simultaneous and successive inoculations of yeasts and lactic acid bacteria on the fermentation of an unsulfited Tannat grape must. Braz J Microbiol 45:59-66.

Nehme N, Mathieu F and Taillandier P. 2010. Impact of the co-culture of Saccharomyces cerevisiae-Oenococcus oeni on malolactic fermentation and partial characterization of a yeast-derived inhibitory peptidic fraction. Food Microbiol 27:150-157.

Ortega-Heras M, Pérez-Magariño S, Del-Villar-Garrachón V, GonzálezHuerta C, Moro González LC, Guadarrama Rodríguez A, Villanueva Sánchez S, Gallo González R and Martín de la Helguera S. 2014. Study of the effect of vintage, maturity degree, and irrigation on the amino acid and biogenic amine content of a white wine from Verdejo variety. J Sci Food Agric 94:2073-2082.
Özogul Y and Özogul F. 2019. Chapter 1: Biogenic amines formation, toxicity, regulations in food. In Biogenic Amines in Food: Analysis, Occurrence and Toxicity. Saad B and Tofalo R (eds.), pp. 1-17. Royal Society of Chemistry, London.

Rodríguez-Bencomo JJ and Mehdi A. 2019. Removal of biogenic amines from hydroalcoholic solutions by functionalized silica. Food Chem 297:125027.

Ruiz P, Izquierdo PM, Seseña S and Palop ML. 2010. Selection of autochthonous Oenococcus oeni strains according to their oenological properties and vinification results. Int J Food Microbiol 137:230-235.

Smit AY and du Toit M. 2013. Evaluating the influence of malolactic fermentation inoculation practices and ageing on lees on biogenic amine production in wine. Food Bioprocess Technol 6:198-206.

Smit AY, du Toit WJ and du Toit M. 2008. Biogenic amines in wine: Understanding the headache. S Afr J Enol Vitic 29:109-127.

Soufleros E, Barrios M and Bertrand A. 1998. Correlation between the content of biogenic amines and other wine compounds. Am J Enol Vitic 49:266-278

Straub BW, Kicherer M, Schilcher SM and Hammes WP. 1995. The formation of biogenic amines by fermentation organisms. Z LebensmUnters-Forsch 201:79-82.

Tomera JF. 1999. Current knowledge of the health benefits and disadvantages of wine composition. Trends Food Sci Technol 10:129-138.

Zapparoli G, Tosi E, Azzolini M, Vagnoli P and Krieger S. 2009. Bacterial inoculation strategies for the achievement of malolactic fermentation in high alcohol wines. S Afr J Enol Vitic 30:49-55.

Zimatkin SM and Anichtchik OV. 1999. Alcohol-histamine interactions. Alcohol Alcohol 34:141-147.

Zúñiga M, Pardo I and Ferrer S. 1993. An improved medium for distinguishing between homofermentative and heterofermentative lactic acid bacteria. Int J Food Microbiol 18:37-42. 